
creativecommons.org/licenses/by/4.0/), which permits unrestricted re-use, distribution, and reproduction in any medium, provided the original work is properly cited.

\title{
Molecular mechanisms relating to amino acid regulation of protein synthesis
}

\author{
Yangchun $\mathrm{CaO}^{1}$ (1) , Shimin $\mathrm{Liu}^{2}$, Kai Liu ${ }^{1}$, Imtiaz Hussain Raja Abbasi ${ }^{1,3}$, Chuanjiang Cai ${ }^{1}$ and Junhu Yao ${ }^{1 *}$ \\ ${ }^{1}$ College of Animal Science and Technology, Northwest A\&F University, Yangling Shaanxi, 712100, People's Republic of China \\ ${ }^{2}$ Institute of Agriculture, University of Western Australia, Crawley, WA 6009, Australia \\ ${ }^{3}$ Department of Animal Nutrition, Faculty of Animal Production and Technology, Cholistan University of Veterinary \\ and Animal Sciences, Bahawalpur, Punjab, Pakistan
}

\begin{abstract}
Some amino acids (AA) act through several signalling pathways and mechanisms to mediate the control of gene expression at the translation level, and the regulation occurs, specifically, on the initiation and the signalling pathways for translation. The translation of mRNA to protein synthesis proceeds through the steps of initiation and elongation, and AA act as important feed-forward activators that are involved in many pathways, such as the sensing and the transportation of AA by cells, in these steps in many tissues of mammals. For the translation, phosphorylation of eukaryotic translation initiation factor $2 \alpha(\mathrm{eIF} 2 \alpha)$ is a critical molecule that controls the translation initiation and its functions can be regulated by some AA. Another control point in the mRNA binding step in the translation initiation is at the regulation by mammalian target of rapamycin, which requires a change of phosphorylation status of ribosomal protein S6. In fact, the change of phosphorylation status of ribosomal protein $\mathrm{S} 6$ might be involved in global protein synthesis. The present review summarises recent work on the molecular mechanisms of the regulation of protein synthesis by AA and highlights new findings.
\end{abstract}

Key words: Amino acids: Mammalian target of rapamycin (mTOR): eIF2: eEF2: Protein synthesis

\section{Introduction}

Amino acids (AA) are the physical basis of life as the substrates of protein synthesis in all tissues of animals. AA are divided into the essential, which cannot be synthesised by animals per se or the synthesis does not adequately meet the animal's demand, or the non-essential for animals. The essential AA must be obtained in food and are comprised of arginine (Arg), cysteine (Cys), histidine (His), isoleucine (Ile), leucine (Leu), lysine (Lys), methionine (Met), phenylalanine (Phe), proline (Pro), threonine (Thr), tryptophan (Trp), tyrosine (Tyr) and valine (Val). The remaining AA are non-essential. Some essential AA can be classified as functional AA, and they not only serve as substrates for protein synthesis, but also function as signal molecules and participate in the regulation of metabolism, such as protein synthesis, of the body.

The synthesis of protein in tissues consists of sequential steps and consumes energy. The process of mRNA translation requires a substantial amount of cellular materials ${ }^{(1)}$, and a sufficient supply of AA is a prerequisite for maintaining the optimal rate of protein synthesis ${ }^{(2)}$. These rate-limiting steps of biosynthetic processes are usually considered as the efficiency targets for regulatory mechanisms to control the processes ${ }^{(3)}$. For protein synthesis, the limiting steps are the initiation and elongation of a peptide chain. Increasing evidence has shown that some AA acts also as signalling molecules to change the phosphorylation status, then the functions of several proteins involved in the regulation of mRNA translation. Understanding these relationships between the functional AA and their regulation on mRNA translation could provide a base to develop nutritional approaches to improve protein synthesis in animal production.

There are two regulatory steps that limit the initiation of mRNA translation: one is the assembly of a ternary complex including eukaryotic initiation factors (eIF) 2 and Met-tRNA (Fig. 1), and the other is the assembly of the eIF 4F (eIF4F) complex in the mammalian target of rapamycin (mTOR) pathway (Fig. 2) ${ }^{(2,3)}$. The ternary complex is composed of eIF2, GTP and Met-tRNA. The eIF4F complex contains three initiation factors: eIF4A, eIF4E and eIF $4 G^{(4)}$. After the complement of the assembly, both complexes bind to $40 \mathrm{~S}$ ribosomal subunits ${ }^{(5)}$, which then scan toward the start codon and polypeptide formation begins. As for the elongation of peptide, the process is regulated by the activity of eukaryotic elongation factors (eEF). The initiation and elongation of protein synthesis can be affected by $\mathrm{AA}$ and several growth factors. The objective of the present

\footnotetext{
Abbreviations: 4EBP1, inhibitory 4E-binding protein-1; AA, amino acid; Arg, arginine; eEF, eukaryotic translation elongation factor; eIF, eukaryotic initiation factor; GCN2, general control nonderepressible 2; Ile, isoleucine; Leu, leucine; MAP4K3, mitogen-activated protein kinase 3; Met, methionine; mTOR, mammalian target of rapamycin; mTORC1, mammalian target of rapamycin complex 1; mTORC2, mammalian target of rapamycin complex 2; Rag, Ras-related GTP-binding protein; Rheb, Ras homologue enriched in brain; S6K1, S6 kinase 1; Thr, threonine; Trp, tryptophan; TSC, tuberous sclerosis complex; Val, valine; Vps34, vacuolar protein sorting 34 .
}

* Corresponding author: Junhu Yao, fax +862987092164, email yaojunhu2004@sohu.com 


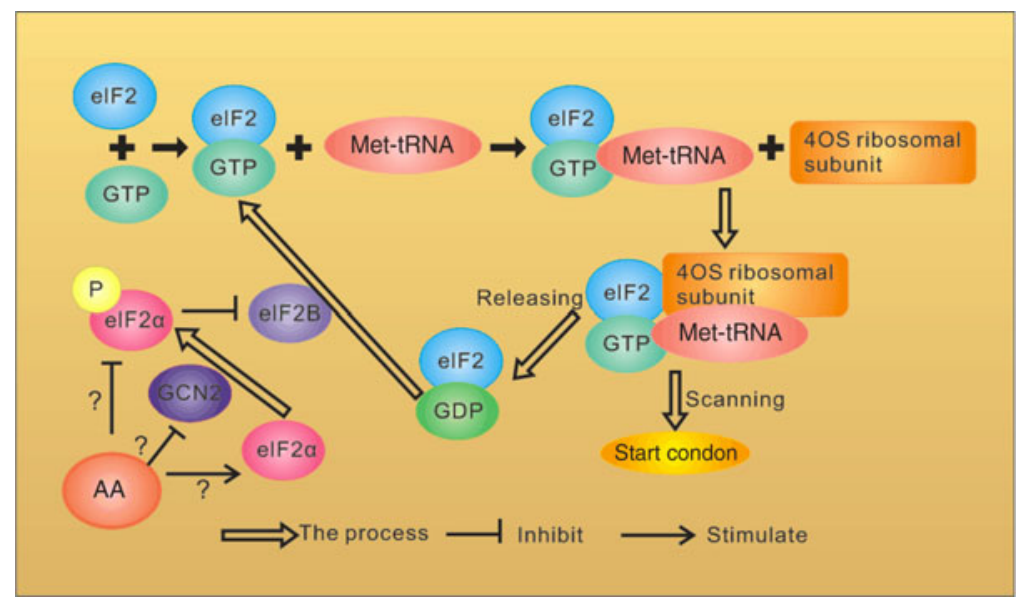

Fig. 1. Regulation of the assembly of the ternary complex by amino acids. elF2, eukaryotic initiation factor-2; Met, methionine;?, unknown interactions between these factors; GCN2, general control nonderepressible 2; AA, amino acids. For a colour figure, see the online version of the paper.

review was to review the molecular mechanisms relating to AA regulation of protein synthesis, with particular focus on the effects of AA on the key signal pathways for the process of the protein translation initiation and elongation.

\section{Amino acids regulate the assembly of the ternary complex}

As illustrated in Fig. 1, the assembly of the ternary complex is a cyclic process starting from the binding of eIF2 to GTP and Met-tRNA, followed by the shift of the complex to $40 \mathrm{~S}$ ribosomal subunits $^{(2,5)}$. When the start codon is recognised, active eIF2GTP is converted to the inactive binary complex, eIF2-GDP, which is released to the cytoplasm. However, the assembly of the ternary complex requires more GTP-eIF2. The guanine nucleotide exchange reaction is mediated by eIF2B, which can be inactivated by the phosphorylation of eIF $2 \alpha$ (subunit targeted for a few serine kinases that phosphorylate serine) through sequestering eIF2B from GDP as a competitive inhibitor ${ }^{(6)}$.

As eIF2 promotes the binding of the initiator Met-tRNA to $40 \mathrm{~S}$ ribosomal subunits, the activity of which can be regulated by phosphorylation of itself, the phosphorylation status of eIF $2 \alpha$ thus plays an important role in controlling the translation initiation, and this status can be regulated by some $\mathrm{AA}^{(5,7)}$. Studies have shown that deprivation of AA enhanced eIF2 $\alpha$ phosphorylation and inhibition of eIF2B activity, resulting in a decrease of protein synthesis in Ehrlich cells ${ }^{(8,9)}$. The eIF $2 \alpha$ kinase, general control nonderepressible 2 (GCN2), has been identified to induce phosphorylation of eIF $2 \alpha$ in mouse embryonic stem cells ${ }^{(10,11)}$ and can be activated by deprivation of $\mathrm{AA}^{(12)}$. In addition, a minor increase in eIF2 $\alpha$ phosphorylation was observed in CHO K1 cells when AA was withdrawn, suggesting that mammalian GCN2 may be activated over a period of AA withdrawal ${ }^{(7)}$, presumably through the accumulation of uncharged tRNA ${ }^{(13)}$.

Despite a clear role of eIF $2 \alpha$ phosphorylation in the regulation of protein synthesis by AA in vitro, results from animal studies in vivo have been less definitive ${ }^{(2)}$. When rats were fasted for $18 \mathrm{~h}$ and then fed a protein-containing meal, neither eIF $2 \alpha$ phosphorylation nor eIF2B activity was affected by the meal in both the liver and skeletal muscle, although protein synthesis was increased by supply of the meal ${ }^{(14)}$. Oral administration of Leu increased protein synthesis in the skeletal muscle in food-deprived rats without causing any change in eIF2 $\alpha$ phosphorylation or eIF2B activity ${ }^{(15)}$. However, in the livers of food-deprived young rats, lack of Trp, Leu or a mix of Leu, Ile and Val resulted in both an increase in eIF2 $\alpha$ phosphorylation and an inhibition of eIF2B activity ${ }^{(16)}$. Recent results indicate that AA deprivation can regulate eIF2B activity independently of changes in eIF $2 \alpha$ phosphorylation, probably via changes in phosphorylation of eIF2B itself ${ }^{(17)}$. Another study showed that the addition of Ile, Leu, Met, and Thr together had no effect on eIF $2 \alpha$ phosphorylation in mammary tissue of dairy cows ${ }^{(18)}$. Besides, removal of individual AA from the medium had no effect on eIF $2 \alpha$ phosphorylation in mammary tissue slices and MAC-T cells, whereas depletion of all the essential AA increased eIF $2 \alpha$ phosphorylation ${ }^{(19,20)}$. Also, the addition of histidine, but not Leu or Met plus Lys reduced eIF2 $\alpha$ phosphorylation in mammary glands of feed-deprived lactating cows ${ }^{(21)}$. Thus, AA affect protein synthesis through the regulation of the phosphorylation status of eIF2, as well as eIF2B.

Mechanism of regulating of binding proteins, mammalian target of rapamycin action and translation pathway

An important mechanism by which mTOR activity can be moderated is through interactions with other proteins. mTOR is a highly conserved protein kinase that regulates the growth and metabolism of mammals. The way mTOR regulates protein synthesis has been well described ${ }^{(3,22)}$, as shown in Fig. 2. mTOR plays an important role in the second regulated step in the translation initiation involving the binding of mRNA to $40 \mathrm{~S}$ ribosomal subunits, a reaction mediated by a triad of initiation factors collectively referred to as eIF $4 \mathrm{~F}^{(23)}$. The eIF4F complex is composed of three proteins: eIF4A, eIF4E (an RNA helicase that binds to the mGTP cap present at the $5^{\prime}$ end of mRNA) ${ }^{(7)}$ and eIF4G (a scaffolding protein that binds not only to eIF4A and eIF4E, but also to eIF3, which is bound to $40 \mathrm{~S}$ ribosomal subunits) ${ }^{(2)}$. Thus, mRNA binds to $40 \mathrm{~S}$ ribosomal subunits through the association of the eIF4F-mRNA complex with the eIF3-40S ribosomal subunit complex. The inhibitory 4E-binding protein-1 (4EBP1; also known 
Fig. 2. Amino acid (AA) regulation of the assembly of eukaryotic initiation factor-4F (elF4F) in the mammalian target of rapamycin (mTOR) pathway. 4EBP1, inhibitory $4 \mathrm{E}$-binding protein-1;?, uncertain of whether AA regulate phosphorylation of $4 \mathrm{EBP} 1$ directly or indirectly through mTOR. For a colour figure, see the online version of the paper.

as eIF4EBP1) inhibits eIF4G binding to $\operatorname{eIF} \mathrm{E}^{(3)}$ and the signal transduction-mediated phosphorylation of 4EBP1 leads to its dissociation from eIF4E, allowing the recruitment of eIF $4 \mathrm{G}$ and eIF4A $\mathrm{A}^{(24)}$

Studies have shown that AA affect the phosphorylation of 4EBP1. When HEK293 cells and CHO-IR cells were cultured in the media added with a mixture of AA, or individual AA, each of the essential AA was partially effective in promoting 4EBP1 phosphorylation and among them, Leu was the most potent one ${ }^{(25,26)}$. In vivo experiments show similar results. In neonatal piglets ${ }^{(27)}$ and rats ${ }^{(14,15,28)}$ subjected to overnight food deprivation, rates of protein synthesis were reduced in skeletal muscles and the liver, and feeding either a complete meal or a meal consisting of protein only rapidly reversed the inhibition, associated with enhanced phosphorylation of 4EBP1, dissociation of eIF4E from the inactive 4EBP1-eIF4E complex, and assembly of the active eIF4G-eIF4E complex.

\section{Mode of action of mammalian target of rapamycin complex 1 and mammalian target of rapamycin complex 2}

mTOR exists in two distinct multiprotein complexes, mTOR complex 1 (mTORC1) and mTOR complex 2 (mTORC2), which are known to differ in their subunit compositions, sensitivity to rapamycin and cell signalling activity ${ }^{(29,30)}$. Both complexes contain mTOR and mammalian lethal SEC13 protein 8; however, they are different in that mTORC1 contains the regulatoryassociated protein of mTOR (raptor), whereas mTORC2 contains rapamycin-associated protein of mTOR (rapamycin-insensitive companion of TOR, or rictor) ${ }^{(31)}$. With these differences present, mTORC1 is highly sensitive to rapamycin but mTORC2 is not. The mTORC1 activity is regulated by modulation of tumour suppressor tuberous sclerosis complex $1 / 2$ (TSC $1 / 2)$ activity $^{(30,31)}$.
This complex is an active dimer that is purposed to limit mTORC1 signalling through the activity of GTPase. These proteins function to split a phosphate from GTP to yield GDP. Therefore, TSC $1 / 2$ is known to negatively regulate mTORC1 activity by converting the GTPase protein Rheb into its inactive GDP-bound state ${ }^{(30,32)}$. Thus, the GTPase activity of Rheb is highly governed by TSC $1 / 2$. Moreover, when Rheb is in its active GTP-bound state, it translocates to the lysosome where mTORC1 is activated ${ }^{(31)}$

The mTORC1 pathway regulates cell growth in response to numerous cues, including AA, which promotes mTORC1 translocation to the lysosomal surface, its site of activation. The heterodimeric Ras-related GTP-binding protein (Rag)A/BRagC/D GTPases, the regulator complex that tethers Rags to the lysosome, and the vacuolar-type $\mathrm{H}^{+}$-adenosine triphosphatase form a signalling system that is necessary for AA sensing by mTORC1. However, hepatitis $\mathrm{B}$ virus $\mathrm{X}$-interacting protein and $\mathrm{C} 7$ or $\mathrm{f} 59$ as two additional regulator components form a pentameric complex that is essential for localising Rag GTPases to the lysosomal surface and activating mTORC1 in response to $\mathrm{AA}$. In addition, to be a scaffold, the regulator promotes nucleotide exchange of RagB and of the highly related Rag $\mathrm{A}^{(33)}$. Rapamycin-insensitive mTORC1 phosphorylates and activates cell growth regulators S6 kinase 1 (S6K1) and S6 kinase 2 (S6K2) and activates ribosomal protein S6 ${ }^{(34)}$, and it phosphorylates and inactivates the translational repressors eukaryotic translation initiation factor 4EBP1; further, 4EBP1 targets and prevents eIF4E-eIF4G interaction and thus inhibits translation initiation $^{(35)}$. mTOR inhibitor rapamycin dephosphorylates S6K1 and 4EBP1, leading to G1 growth arrest and inhibition of ribosome biosynthesis and autophagy ${ }^{(36,37)}$. The anti-tumour activity of rapamycin as a single agent has been described in vitro and in vivo ${ }^{(38,39)}$. Furthermore, mTORC2 activates protein kinase $\mathrm{B}$ (PKB, also known as Akt) and regulates the actin 
cytoskeleton $^{(40,41)}$. Rapamycin dephosphorylates the mTORC2 component rictor $^{(42)}$. Although rictor phosphorylation may affect Akt phosphorylation, the biological consequence of its phosphorylation is relatively unknown ${ }^{(43)}$. Both mTORC1 and mTORC2 respond to hormones and growth factors ${ }^{(44)}$; however, only mTORC1 has been proposed to be acutely regulated by nutrients such as $\mathrm{AA}^{(45,46)}$. Many reports have suggested that AA activate mTORC1, increase cell growth through increased ribosome biogenesis and protein biosynthesis and suppression of autophagy ${ }^{(4,47)}$. Among AA, Leu is in the primacy as discussed below. Growth factor signals have little or no effect on mTORC1 signalling in the absence of $\mathrm{AA}^{(25)}$.

Target of rapamycin interrelating proteins and regulation pathway of mammalian target of rapamycin signalling

Recent studies have shown that AA regulate phosphorylation of $4 \mathrm{EBP} 1$ indirectly, and three new proteins have been revealed to mediate the regulatory effects on protein synthesis by AA, including the Ragulator-Rag complex, vacuolar protein sorting 34 (Vps34) and mitogen-activated protein kinase 3 (MAP4K3). Then mTORC1 regulates cell growth by coordinating upstream signals from growth factors, intracellular energy levels, and AA availability $^{(48)}$. The tuberous suppressor complex, TSC1/TSC2, affects the activity of mTORC1 by regulating the GTP-loading state of Rheb (Ras homologue enriched in brain), a Rheb-related GTP binding protein, and GTP-Rheb interacts with and activates mTORC1 $^{(49)}$. However, TSC1/TSC2 is dispensable for the regulation of mTORC1 by AA, and in cells lacking TSC2 the mTORC1 pathway is sensitive to AA starvation but resistant to growth factor withdrawal ${ }^{(50,51)}$. Recent studies have shown that Rag GTPases, which are also members of the Ras family of GTPbinding proteins, are AA-specific regulators in the mTORC1 pathway ${ }^{(52,53)}$. Mammals express four Rag proteins (RagA, B, C and $\mathrm{D}$ ), which form heterodimers consisting of RagA or RagB with RagC or RagD. RagA and RagB, as well as RagC and RagD, are highly like each other and are functionally redundant ${ }^{(53,54)}$. Further, GTP-RagB interacts with mTORC1 by binding to raptor (regulatory-associated protein of $\mathrm{mTOR}$ ), one part of the mTORC1 complex ${ }^{(55)}$, and AA induce the mTORC1-Rag interaction by promoting the loading of RagB with $\mathrm{GTP}^{(53)}$. The activation of the mTORC1 pathway by AA correlates with the movement of mTORC1 from an undefined location to a compartment containing Rab7 $7^{(53)}$, a marker of both late endosomes and lysosomes ${ }^{(56,57)}$. Sancak et al. ${ }^{(53)}$ pointed out that AA stimulate the translocation of mTORC1 to the lysosomal surface, where the Rag GTPases reside irrespective of their GTPloaded states or AA availability, and the translocation of mTORC1 to lysosomes does not depend on growth factors, Rheb or mTORC1 activity ${ }^{(58)}$. Moreover, a trimeric regulator complex, including MP1, p14, and p18, is mostly found to interact with Rag GTPases and colocalises with them on lysosomal membranes and is necessary for the AA-dependent recruitment and activation of mTORC1 (Fig. 3) ${ }^{(58,59)}$. In addition to the Ragulator-Rag complex, other components have also been implicated in the upstream control of mTORC1 by AA ${ }^{(17)}$. One of them is a homologue of the mammalian protein kinase,
MAP4K3, which is identified as a nutrient-sensitive regulator of mTOR signalling by a recent RNA interference screen in Drosophila cells ${ }^{(60)}$.

Another possible mediator of AA signalling to mTOR is the class III phosphotidyl-inositol 3-kinase $\operatorname{mVps} 34^{(61,62)}$. AA induce an extracellular $\mathrm{Ca}$ influx that activates calmodulin, which in turn binds to and activates $\mathrm{mVps} 34$, and $\mathrm{mVps} 34$ then produces phosphatidylinositol-3-phosphate (PI3P) that somehow activates mTORC1. The mechanism also involves the formation of a calmodulin-mVps34-mTORC1 super-complex ${ }^{(62)}$. Additionally, Vps34 has also been reported to regulate Rab7-mediated vesicular trafficking ${ }^{(63,64)}$. The product of hVps34 and PI3P is involved in recruiting proteins to endosomal membranes containing FYVE or phox homology (PX) domains ${ }^{(65)}$, with these PI3P-rich microdomains serving as platforms to build signalling complexes ${ }^{(66,67)}$. Given the Rag-dependent re-localisation of mTORC1 to Rag7positive structures, this connection could provide a potential explanation for how Vps34 depletion could interfere with the AA control of mTORC1 activity ${ }^{(60)}$. Recent studies have revealed the functions of class III PI3 kinase in murine myotubes ${ }^{(68)}$, HepG2, and CHO cells ${ }^{(63)}$. In states of AA starvation, hVps34 demonstrated reduced function. However, the functions restored with re-addition of $\mathrm{AA}^{(62,63)}$. Further, for the first time, mTOR signalling was enhanced in response to an acute stimulation with the proteinogenic AA, Leu, in cultured human myotubes, and hVps34 served as a nutrient-sensing protein for mTOR signalling mediated by $\mathrm{AA}^{(69)}$. Moreover, hVps34 plays an unappreciated role in nutrient sensing and activation of ribosomal protein $\mathrm{S} 6$ kinase $\beta-1$ (S6K1) and is a component of the AA-regulated arms of S6K1 regulation ${ }^{(67)}$. Many advances have been made to illuminate the mechanism by which AA regulate mTORC1. The Rag complex, MAP4K3 and mVps34 have been implicated. Some studies showed that MAP4K3 converged on the signalling through mTOR in mammalian cells by activating proteins Rag 1-4 and class III phosphatidylinositol $3^{\prime}$-kinase $\mathrm{Vps} 34^{(53,61,62)}$. A recent study suggests that it is the activity of $\mathrm{Vps} 34$ that is the primary modulator of the Leu-stimulated mTOR signalling in rat muscle ${ }^{(67)}$, which is different from the interaction between MAP4K3 and Vps34 as described previously. More studies should be done to verify where these proteins fit into the AA regulatory pathway.

\section{Amino acids regulate the elongation process of protein synthesis}

Ribosomes join AA together in the sequence determined by the mRNA to make the corresponding protein, and AA are brought onto ribosomes attached to tRNA. Further elongation requires the help of elongation factor proteins and tRNA with attached AA (called aminoacyl tRNA) that are brought onto ribosomes by one such elongation factor ${ }^{(18)}$. This factor is termed EF-Tu in prokaryotes and EF1 in eukaryotes. To complete the round of elongation, a second elongation factor, called EF-G in prokaryotes and eukaryotic elongation factor 2 in eukaryotes, is needed. However, ribosomes have three aminoacyl tRNA binding sites: the acceptor site (A), the peptidyl site (P) and the exit site $(\mathrm{E})^{(70)}$. This elongation factor moves tRNA such that the spent tRNA that has lost the protein chain moves fully into the E site, 


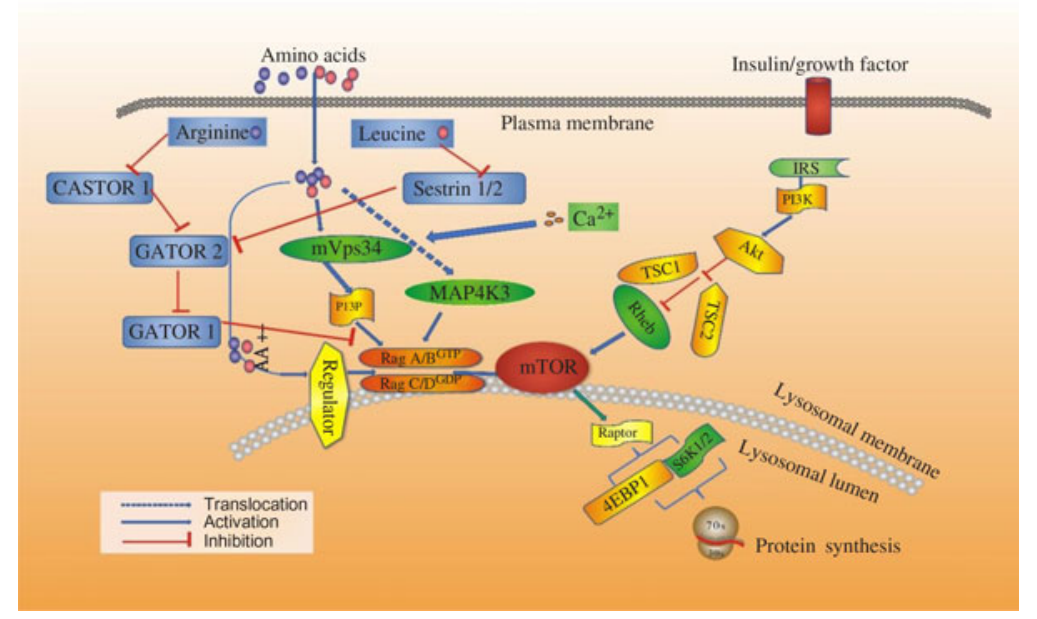

Fig. 3. Signalling by amino acids (AA) and other factors, target of rapamycin (TOR) interrelating protein activation and mammalian target of rapamycin ( $m$ TOR) signalling for protein synthesis. 4EBP1, inhibitory 4E-binding protein-1; Akt, protein kinase B; IRS, insulin receptors; MAP4K3, mitogen-activated protein kinase-3; PI3K, phosphatidylinositol-3-kinases; PI3P, phosphatidylinositol-3-phosphate; Rag, Ras-related GTP-binding protein; Rheb, Ras homologue enriched in brain; S6K1, ribosomal protein S6 kinase $\beta-1 ;$ TSC1/TSC2, tuberous suppressor complex; Vps34, vacuolar protein sorting-34. For a colour figure, see the online version of the paper.

and tRNA with the growing protein chain moves fully into the $\mathrm{P}$ site. The mRNA is also shifted over one codon by EF-G so that the next codon is in the A site. The A site is now empty of tRNA and the next aminoacyl tRNA can be brought into it.

AA modulate the translation elongation machinery through the control of the activity of eEF2. The eEF2 is subject to phosphorylation, and the phosphorylated form of the protein is inactive in translation ${ }^{(70)}$. AA depletion can result in increased phosphorylation of $\mathrm{eEF}^{(26)}$. In addition, Arriola Apelo et al. ${ }^{(18)}$ revealed the effects of Leu, Ile, Met and Thr and their interactions on the activity of eEF2 in mammary tissue. According to this study, eEF2 phosphorylation was linearly decreased by Ile and Leu, Thr caused a curvilinear decrease in eEF2 phosphorylation, and both Ile and Leu negatively interacted on eEF2, and Thr tended to inhibit the Leu effects on eEF2. Furthermore, mTORC1 could control the phosphorylation of eEF2 ${ }^{(17)}$, and the phosphorylation of eEF2 by eEF2K, a calmodulin-dependent kinase downstream of mTOR and S6K1, also inhibits its translation elongation $\operatorname{activity}^{(18)}$.

Arginine and leucine in the primacy on mammalian target of rapamycin complex 1 signalling and synthesis of protein

Although several AA can regulate mTORC1 signalling through the Rag GTPase, Arg and Leu are the best established and deprivation of either strongly inhibits mTORC1 in various cell types ${ }^{(25)}$. A complex which is called GATOR can interact with the Rags and is composed of two subcomplexes (GATOR1 and GATOR2). Inhibition of GATOR1 subunits makes mTORC1 signalling resistant to AA deprivation, while inhibition of GATOR2 subunits suppresses mTORC1 signalling ${ }^{(71)}$. The Leu-binding capacity of Sestrin2 is required for Leu to activate mTORC1 in cells. This indicates that Sestrin2 is a Leu sensor for the mTORC1 pathway ${ }^{(72)}$. However, stimulation by Arg in cells to activate mTORC1 is not well understood. Moreover,
CASTOR1 works as an Arg sensor for the mTORC1 pathway and interacts with GATOR2 and is required for Arg deprivation to inhibit TTORC1 $^{(73)}$. CASTOR1 homodimerises and can also heterodimerise with the related protein CASTOR2 ${ }^{(73)}$. Arg disrupts the CASTOR1-GATOR2 complex by binding to CASTOR1. The proteins that sense AA and signal to the Rag GTPases were indefinable until recently. Sestrin2 as a cytosolic Leu sensor and solute carrier family 38 member 9 (SLC38A9) as a putative lysosomal Arg sensor for the mTORC1 pathway was identified $^{(72,74)}$. While Sestrin2 interacts with GATOR2 to inhibit mTORC1 signalling in the absence of Leu, SLC38A9 forms a super complex with a regulator and is necessary for transmitting Arg, but not Leu, sufficiency to mTORC1 $1^{(72,74,75)}$. For Leu to activate mTORC1, Sestrin2 must be able to bind Leu and work as a Leu sensor for the mTORC1 pathway. Furthermore, Sestrin2 has an appreciable affinity for Met. It would not be surprising if, in contexts where Leu concentrations are low and those of Met are high, Sestrin 2 may serve as a Met sensor for the mTORC1 pathway (Fig. 3) ${ }^{(72,75)}$.

The primacy of Leu on mTORC1 signalling and the effect on protein synthesis is of great interest, nutritionally both in terms of the reduction of loss of lean body mass in various disease states and in the maintenance/enhancement of lean body mass in the healthy condition. Specifically, Leu has been reported to activate the mTOR/S6K1 signalling pathway ${ }^{(76-78)}$. Leu, acting through an mTORC1-dependent pathway, stimulates the translation of specific mRNA both by increasing availability of eIF4E and by stimulating phosphorylation of $S 6^{(79)}$. In addition, Leu induces cell migration and Rac activation controlling cytoskeletal reorganisation through $\mathrm{mTORC} 2^{(80)}$. However, deprivation of Leu leads to a cellular response overlapping with but distinct from mTOR inhibition ${ }^{(81)}$, and deprivation and rapamycin have been proposed to have synergistic effects on protein synthesis $^{(82)}$.

The synthesis of protein in tissues is rapidly stimulated after oral intake of nutrients, and Leu appears to mediate most of the effective AA on protein metabolism. The central role of Leu 
mediating the anabolic effects of protein/AA on muscle protein metabolism was noted from the mid-1970s ${ }^{(83,84)}$. Not only does Leu stimulate protein synthesis in skeletal muscle, but also affects protein degradation in skeletal muscle and the liver, through TORC1-dependent and independent mechanisms. Protein synthesis is associated with an increase in 4EBP1 phosphorylation, a decrease in association of 4EBP1 with eIF4E, an increase in association of eIF4G with eIF4E, and an increase in S6K1 phosphorylation. Long et al. ${ }^{(85)}$ showed that binding of Rheb to mTOR was promoted by the presence of AA and inhibited by the withdrawal of a mixture of AA or Leu only, but it seemed unlikely that this mechanism could account for the specific physiological effects of Leu in intact animals. Another unique signalling function of Leu in the regulation of metabolism, in part through the regulation of mTOR signalling, has been extensively supported by studies in vivo, primarily in rodents ${ }^{(6)}$ and in human subjects $^{(86,87)}$.

Leu also acts on the central nervous system to control overall food intake through TORC1, and food selection probably through GCN2 ${ }^{(88,89)}$. Direct administration of 1-Leu (but not l-Val) near the arcuate nucleus region in rat hypothalamus stimulates hypothalamic TOR signalling and results in decreased food intake (anorexia) and significant weight loss ${ }^{(90)}$. Activation of GCN2 occurs in response to a deficiency of any essential AA; however, the regulation of mTORC1 is most responsive to specific individual $\mathrm{AA}^{(6)}$. Among AA, Leu and Arg share nearly comparable inhibitory effects ${ }^{(91)}$ but underlying mechanisms are puzzling; other than their participation in polypeptide chain elongation through the mediation of six tRNA, these two AA have no commonalities in their transport or metabolism-regulatory functions. Additionally, it should be mentioned that alterations in the extracellular concentration of AA whose transport is $\mathrm{Na}^{+}$linked, for example, glutamine but (not Leu), will lead to parallel alterations in cell hydration; cell swelling induced by any mechanism is accompanied by activation of several anabolic pathways, including mTORC1, whereas cell dehydration is inhibitory $^{(92,93)}$.

Leu also interacts with carbohydrate on metabolism in cells and the body. Leu plus carbohydrate have the same effect as Leu alone, and administration of either Ile or Val alone had no effect and administration of Leu produced only a slight, transient rise in plasma insulin concentration at $30 \mathrm{~min}$, whereas carbohydrate caused a much greater increase that was maintained at 60 $\min ^{(94)}$. Using a meal-feeding model, Lynch et al. ${ }^{(95)}$ confirmed the stimulatory effect of Leu on tissue protein synthesis in gastrocnemius, kidney and adipose tissue, whereas a carbohydrate meal had no effect despite a robust increase in plasma insulin. Further, Leu also promotes leptin synthesis in the adipocyte ${ }^{(96)}$, and both Leu and leptin action on leptin-sensitive neurons, signals that reflect immediate and long-term adequate nutrition, activate TORC1 to suppress further food intake. The stimulation of TORC1 by Leu appears to be initiated at an intracellular site. In Xenopus oocytes, extracellular Leu is unable to promote S6K1 phosphorylation, but expression of a recombinant system L transporter confers responsiveness to extracellular Leu and direct intracytoplasmic injection of 1-Leu (or Trp, Arg, Lys and glycine, but not d-Leu, alanine, Pro, glutamate or glutamine) stimulates S6K1-P in a rapamycin-sensitive manner ${ }^{(97)}$.
Leu and Phe both promoted synthesis or regulated secretion of $\alpha$-amylase secretion in dairy goats ${ }^{(98)}$. Phe also regulates the synthesis of trypsin and lipase through mRNA translation initiation factors S6K1 and 4EBP1 ${ }^{(99)}$. The branched-chain AA Val, Leu, and Ile are essential AA; among these, Leu particularly stimulates protein synthesis ${ }^{(100)}$. It has been found that Leu specifically stimulates phosphotidyl-inositol 3-kinase ${ }^{(101)}$, the kinase for $\mathrm{mTOR}^{(102)}$, resulting in stimulation of the protein synthesis machinery, including ribosomal 56 kinase and $4 \mathrm{E}$ binding protein in skeletal muscles ${ }^{(103)}$. However, suppression of proteolysis by Leu does not involve an mTOR signalling pathway in mouse C2C12 myotubes $^{(82)}$ and isolated rat hepatocytes ${ }^{(104)}$. The suppression of myofibrillar proteolysis by Leu does not involve the mTOR signalling pathway in chick myotubes cultured in vitro, and oral administration of Leu suppresses myofibrillar proteolysis in chicks in vivo ${ }^{(105)}$.

\section{Conclusion}

AA play a key role in both maintaining body metabolism and signalling protein synthesis. Several new proteins have been found in the AA-regulating pathways, and their regulatory mechanisms remain unclear. Future studies will need to depict these mechanisms because of their critical roles in modulating protein synthesis and other metabolism in the body.

\section{Author ORCID}

Yangchun Cao http://orcid.org/0000-0003-1033-2909

\section{Acknowledgements}

The present review was supported by the National Natural Science Foundation of China (grant no. 31472122 and 31672451) and the Collaborative Innovation Major Project of Industry, University, Research, and Application in the Yangling Demonstration Zone (grant no. 2016CXY-18). The paper represents the original work of the authors. Y. C. was responsible for researching, writing and preparing the manuscript. K. L. and I. H. R. A. contributed the figures. S. L., C. C. and J. Y. provided intellectual input, proof reading and corrections. The authors declare that they have no conflicts of interest.

\section{References}

1. Shimizu Y, Inoue A, Tomari Y, et al. (2001) Cell-free translation reconstituted with purified components. Nat Biotech $\mathbf{1 9}$, 751-755.

2. Kimball SR (2002) Regulation of global and specific mRNA translation by amino acids. J Nutr 132, 883-886.

3. Ma XM \& Blenis J (2009) Molecular mechanisms of mTOR mediated translational control. Nat Rev Mol Cell Biol 10, 307-318.

4. Hershey JWB \& Merrick WC (2000) The pathway and mechanism of initiation of protein synthesis. In Translational Control of Gene Expression, pp. 33-88 [N Sonenberg, JWB Hershey and MB Mathews, editors]. Cold Spring Harbor, NY: Cold Spring Harbor Laboratory Press. 
5. Hinnebusch AG (2000) Mechanism and regulation of initiator methionyl-tRNA binding to ribosomes. In Translational Control of Gene Expression, pp. 185-243 [N Sonenberg, JWB Hershey and MB Mathews, editors]. Cold Spring Harbor, NY: Cold Spring Harbor Laboratory Press.

6. Stipanuk MH (2007) Leucine and protein synthesis: mTOR and beyond. Nutr Rev 65, 122-129.

7. Beugnet A, Tee AR, Taylor PM, et al. (2003) Regulation of targets of mTOR (mammalian target of rapamycin) signaling by intracellular amino acid availability. Biochem J 372, 555-566.

8. Scorsone KA, Panniers R, Rowlands AG, et al. (1987) Phosphorylation of eukaryotic initiation factor 2 during physiological stresses which affect protein synthesis. $J$ Biol Chem 262, 14538-14543.

9. Rowlands AG, Montine KS, Henshaw EC, et al. (1988) Physiological stresses inhibit guanine-nucleotide-exchange factor in Ehrlich cells. Eur J Biochem 175, 93-99.

10. Berlanga JJ, Santoyo JD \& Haro C (1999) Characterization of a mammalian homolog of GCN2 eukaryotic initiation factor 2 kinase. Eur J Biochem 265, 754-762.

11. Sood R, Porter AC, Olsen DA, et al. (2000) A mammalian homologue of GCN2 protein kinase important for translational control by phosphorylation of eukaryotic initiation factor- $2 \alpha$. Genetics 154, 787-801.

12. Harding HP, Novoa I, Zhang Y, et al. (2000) Regulated translation initiation controls stress-induced gene expression in mammalian cells. Mol Cell 6, 1099-1108.

13. Hinnebusch AG (1997) Translational regulation of yeast GCN4. J Biol Chem 272, 21661-21664.

14. Yoshizawa F, Kimball SR \& Jefferson LS (1997) Modulation of translation initiation in rat skeletal muscle and liver in response to food intake. Biochem Biophys Res Commun 240, 825-831.

15. Anthony JC, Anthony TG, Kimball SR, et al. (2000) Orally administered leucine stimulates protein synthesis in skeletal muscle of postabsorptive rats in association with the increased eIF4F formation. J Nutr 130, 139-145.

16. Anthony TG, Reiter AK, Anthony JC, et al. (2001) Deficiency of essential dietary amino acids preferentially inhibits mRNA translation of ribosomal proteins in the liver of meal-fed rats. Am J Physiol 281, E430-E439.

17. Proud CG (2007) Amino acids and mTOR signalling in anabolic function. Biochem Soc Trans 35, 1187-1190.

18. Arriola Apelo SI, Singer LM, Lin XY, et al. (2014) Isoleucine, leucine, methionine, and threonine effects on mammalian target of rapamycin signaling in mammary tissue. J Dairy Sci $\mathbf{9}$, 1047-1056.

19. Appuhamy J, Bell A, Nayananjalie W, et al. (2011) Essential amino acids regulate both initiation and elongation of mRNA translation independent of insulin in MAC-T cells and bovine mammary tissue slices. J Nutr 141, 1209-1215.

20. Appuhamy J, Knoebel NA, Nayananjalie W, et al. (2012) Isoleucine and leucine independently regulate mTOR signaling and protein synthesis in MAC-T cells and bovine mammary tissue slices. J Nutr 142, 484-491.

21. Toerien CA, Trout DR \& Cant JP (2010) Nutritional stimulation of milk protein yield of cows is associated with changes in phosphorylation of mammary eukaryotic initiation factor 2 and ribosomal S6 kinase 1. J Nutr 140, 285-292.

22. Pazit P \& Michael NH (2009) mTOR and the control of wholebody metabolism. Cell Biol 21, 209-218.

23. Raught B, Gingras AC \& Sonenberg N (2000) Regulation of ribosomal recruitment in eukaryotes. In Translational Control of Gene Expression, pp. 245-293 [N Sonenberg, JWB Hershey and MB Mathews, editors]. Cold Spring Harbor, NY: Cold Spring Harbor Laboratory Press.
24. Gingras AC, Raught B \& Sonenberg N (1999) eIF4 initiation factors: effectors of mRNA recruitment to ribosomes and regulators of translation. Annu Rev Biochem 68, 913-963.

25. Hara K, Yonezawa K, Weng QP, et al. (1998) Amino acid sufficiency and mTOR regulate p70 S6 kinase and eIF-4E BP1 through a common effector mechanism. $J$ Biol Chem 273, 14484-14494.

26. Wang X, Campbell LE, Miller CM, et al. (1998) Amino acid availability regulates p70 S6 kinase and multiple translation factors. Biochem J 334, 261-267.

27. Davis TA, Nguyen HV, Suryawan A, et al. (2000) Developmental changes in the feeding-induced stimulation of translation initiation in muscle of neonatal pigs. $\mathrm{Am} \mathrm{J}$ Physiol 279, E1226-E1234.

28. Yoshizawa F, Kido T \& Nagasawa T (1999) Stimulative effect of dietary protein on the phosphorylation of p70 S6 kinase in the skeletal muscle and liver of food-deprived rats. Biosci Biotechnol Bioch 63, 1803-1805.

29. Stefano S, Kenneth AD, Stefano C, et al. (2013) Mechanisms regulating skeletal muscle growth and atrophy. FEBS J $\mathbf{2 8 0}$, 4294-4314.

30. Laplante M \& Sabatini DM (2012) mTOR signaling. Cold Spring Harb Perspect Biol 4, a011593.

31. Winter JN, Fox TE, Kester M, et al. (2010) Phosphatidic acid mediates activation of mTORC1 through the ERK signaling pathway. Am J Physiol Cell Physiol 299, C335-C344.

32. Jacobs BL, Goodman CA \& Hornberger TA (2014) The mechanical activation of mTOR signaling: an emerging role for late endosome/lysosomal targeting. J Muscle Res Cell Motil 35, 11-21.

33. Bar-Peled L, Schweitzer LD, Zoncu R, et al. (2012) Ragulator is a GEF for the Rag GTPases that signal amino acid levels to mTORC1. Cell 150, 1196-1208.

34. Holz MK, Ballif BA, Gygi SP, et al. (2005) mTOR and S6K1 mediate assembly of the translation preinitiation complex through dynamic protein interchange and ordered phosphorylation events. Cell 123, 569-580.

35. Richter JD \& Sonenberg N (2005) Regulation of cap-dependent translation by eIF4E inhibitory proteins. Nat $\mathbf{4 3 3}, 477-480$.

36. Easton JB \& Houghton PJ (2006) mTOR and cancer therapy. Oncogene 25, 6436-6446.

37. Inoki K, Ouyang H, Li Y \& Guan KL (2005) Signaling by target of rapamycin proteins in cell growth control. Microbiol Mol Biol Rev 69, 79-100.

38. Dilling MB, Dias P, Shapiro DN, et al. (1994) Rapamycin selectively inhibits the growth of childhood rhabdomyosarcoma cells through inhibition of signaling via the type I insulin-like growth factor receptor. Cancer Res 54, 903-907.

39. Noh WC, Mondesire WH, Peng J, et al. (2004) Determinants of rapamycin sensitivity in breast cancer cells. Clin Cancer Res 10, 1013-1023.

40. Jacinto E, Loewith R, Schmidt A, et al. (2004) Mammalian TOR complex 2 controls the actin cytoskeleton and is rapamycin insensitive. Nat Cell Biol 6, 1122-1128.

41. Sarbassov DD, Ali SM, Kim DH, et al. (2004) A novel binding partner of mTOR, defines a rapamycin-insensitive and raptorindependent pathway that regulates the cytoskeleton. Curr Biol 14, 1296-1302.

42. Akcakanat A, Singh G, Hung MC, et al. (2007) Rapamycin regulates the phosphorylation of rictor. Biochem Biophys Res Commun 362, 330-333.

43. Dibble CC, Asara JM \& Manning BD (2009) Characterization of rictor phosphorylation sites reveals direct regulation of mTOR complex 2 by S6K1. Mol Cell Biol 29, 5657-5670.

44. Wullschleger S, Loewith R \& Hall MN (2006) TOR signaling in growth and metabolism. Cell 124, 471-484. 
45. Dann SG, Selvaraj A \& Thomas G (2007) mTOR Complex1S6K1 signaling: at the crossroads of obesity, diabetes, and cancer. Trends Mol Med 13, 252-259.

46. Huang J, Dibble CC, Matsuzaki M, et al. (2008) The TSC1-TSC2 complex is required for proper activation of mTOR complex 2 . Mol Cell Biol 28, 4104-4115.

47. Kimball SR \& Jefferson LS (2006) New functions for amino acids: effects on gene transcription and translation. $\mathrm{Am} \mathrm{J}$ Clin Nutr 83, 500S-507S.

48. Guertin DA \& Sabatini DM (2007) Defining the role of mTOR in cancer. Cancer Cell 12, 9-22.

49. Laplante M \& Sabatini DM (2009) mTOR signaling at a glance. J Cell Sci 122, 3589-3594.

50. Smith EM, Finn SG, Tee AR, et al. (2005) The tuberous sclerosis protein TSC2 is not required for the regulation of the mammalian target of rapamycin by amino acids and certain cellular stresses. J Biol Chem 280, 18717-18727.

51. Roccio M, Bos JL \& Zwartkruis FJT (2006) Regulation of the small GTPase Rheb by amino acids. Oncogene 25, 657-664.

52. Kim E, Goraksha-Hicks P, Li L, et al. (2008) Regulation of TORC1 by Rag GTPases in nutrient response. Nat Cell Biol 10, 935-945.

53. Sancak Y, Peterson TR, Shaul YD, et al. (2008) The Rag GTPases bind raptor and mediate amino acid signaling to mTORC1. Science 320, 1496-1501.

54. Sekiguchi T, Hirose E, Nakashima NM, et al. (2001) Novel G proteins, Rag $\mathrm{C}$ and Rag D, interact with GTP-binding proteins, Rag A and Rag B. J Biol Chem 276, 7246-7257.

55. Shaw RJ (2008) mTOR signaling: RAG GTPases transmit the amino acid signal. Trends Biochem Sci 33, 565-568.

56. Chavrier P, Parton RG, Hauri HP, et al. (1990) Localization of low molecular weight GTP binding proteins to exocytic and endocytic compartments. Cell 62, 317-329.

57. Luzio JP, Pryor PR \& Bright NA (2007) Lysosomes: fusion and function. Nat Rev Mol Cell Biol 8, 622-632.

58. Yasemin S, Liron BP, Roberto Z, et al. (2010) Ragulator-rag complex targets mTORC1 to the lysosomal surface and is necessary for its activation by amino acids. Cell 141, 290-303.

59. Deldicque L, Theisen D \& Francaux M (2005) Regulation of mTOR by amino acids and resistance exercise in skeletal muscle. Eur J Appl Physiol 94, 1-10.

60. Findlay GM, Yan L, Procter J, et al. (2007) A MAP4 kinase related to Ste20 is a nutrient-sensitive regulator of mTOR signalling. Biochem J 403, 13-20.

61. Byfield MP, Murray JT \& Backer JM (2005) hVps34 is a nutrient-regulated lipid kinase required for activation of p70 S6 kinase. J Biol Chem 280, 33076-33082.

62. Gulati P, Gaspers LD, Dann SG, et al. (2008) Amino acids activate mTOR complex 1 via Ca2+/CaM signaling to hVps34. Cell Metab 7, 456-465.

63. Stein MP, Yan F, Karen LC, et al. (2003) Human VPS34 and p150 are Rab7 interacting partners. Traffic 4, 754-771.

64. Kinchen JM, Kimon D, Johann A, et al. (2008) A pathway for phagosome maturation during engulfment of apoptotic cells. Nat Cell Biol 10, 556-566.

65. Lemmon MA (2003) Phosphoinositide recognition domains. Traffic 4, 201-213.

66. Miaczynska M, Pelkmans L \& Zerial M (2004) Not just a sink: endosomes in control of signal transduction. Curr Opin Cell Biol 16, 400-406.

67. Maya PB, James TM \& Jonathan M (2005) hVps34 is a nutrientregulated lipid kinase required for activation of p70 56 kinase. J Biol Chem 280, 33076-33082.

68. MacKenzie MG, Hamilton DL, Murray JT, et al. (2009) mVps34 is activated following high-resistance contractions. J Physiol 587, 253-260.
69. Petra G \& David CS (2011) The actions of exogenous leucine on mTOR signalling and amino acid transporters in human myotubes. BMC Physiol 11, 10.

70. Carlberg U, Nilsson A \& Nygard O (1990) Functional properties of phosphorylated elongation factor 2. EurJ Biochem 191 , 639-645.

71. Bar-Peled L, Chantranupong L, Cherniack AD, et al. (2013) A tumor suppressor complex with GAP activity for the Rag GTPases that signal amino acid sufficiency to mTORC1. Science 340, 1100-1106.

72. Wolfson RL, Chantranupong L, Saxton RA, et al. (2016) Sestrin2 is a leucine sensor for the mTORC1 pathway. Science 351, 43-48.

73. Chantranupong L, Scaria SM, Saxton RA, et al. (2016). The CASTOR proteins are Arg sensors for the mTORC1 pathway. Cell 165, 153-164.

74. Wang S, Tsun ZY, Wolfson RL, et al. (2015). Lysosomal amino acid transporter SLC38A9 signals Arg sufficiency to mTORC1. Sci 347, 188-194.

75. Chantranupong L, Wolfson RL, Orozco JM, et al. (2014) The Sestrins interact with GATOR2 to negatively regulate the amino-acid-sensing pathway upstream of mTORC1. Cell Rep 9, 1-8.

76. Kimball SR, Shantz LM, Horetsky RL, et al. (1999) Leucine regulates translation of specific mRNAs in L6 myoblasts through mTOR-mediated changes in availability of eIF4E and phosphorylation of ribosomal protein S6. J Biol Chem 274, 11647-11652.

77. Xu G, Kwon G, Marshall CA, et al. (1998) Branched-chain amino acids are essential in the regulation of PHAS-I and p70 S6 kinase by pancreatic $\beta$-cells: a possible role in protein translation and mitogenic signaling. $J$ Biol Chem 273, 28178-28184.

78. Lee MY, Jo SD, Lee JH, et al. (2008) l-Leucine increases [3H]thymidine incorporation in chicken hepatocytes: involvement of the PKC, PI3K/Akt, ERK1/2, and mTOR signaling pathways. J Cell Biochem 105, 1410-1419.

79. Karlsson HK, Nilsson PA, Nilsson J, et al. (2004) Branchedchain amino acids increase p70S6k phosphorylation in human skeletal muscle after resistance exercise. Am J Physiol 287, E1-E7.

80. Hernández-Negrete I, Carretero-Ortega J, Rosenfeldt $\mathrm{H}$, et al. (2007) P-Rex1 links mammalian target of rapamycin signaling to Rac activation and cell migration. $J$ Biol Chem 282, 23708-23715.

81. Peng T, Golub TR \& Sabatini DM (2002) The immunosuppressant rapamycin mimics a starvation-like signal distinct from amino acid and glucose deprivation. Mol Cell Biol 22, 5575-5584.

82. Mordier S, Deval C, Bechet D, et al. (2000) Leucine limitation induces autophagy and activation of lysosome-dependent proteolysis in $\mathrm{C} 2 \mathrm{C} 12$ myotubes through a mammalian target of rapamycin-independent signaling pathway. J Biol Chem 275, 29900-29906.

83. Buse MG \& Reid SS (1975) Leucine. A possible regulator of protein turnover in muscle. J Clin Invest 56, 1250-1261.

84. Tischler ME, Desautels M \& Goldberg AL (1982) Does leucine, leucyl-tRNA, or some metabolite of leucine regulate protein synthesis and degradation in skeletal and cardiac muscle? J Biol Chem 257, 1613-1621.

85. Long X, Ortiz-Vega S, Lin Y, et al. (2005) Rheb binding to mammalian target of rapamycin (mTOR) is regulated by amino acid sufficiency. J Biol Chem 280, 23433-23436.

86. Coffey VG \& Hawley JA (2007) The molecular bases of training adaptation. Sports Med 37, 737-763. 
87. Vary TC \& Lynch CJ (2006) Meal feeding enhances formation of eIF 4F in skeletal muscle: role of increased eIF4E availability and eIF4G phosphorylation. Am J Physiol Endocrinol Metab 290, E631-E642.

88. Hao S, Sharp JW, Ross-Inta CM, et al. (2005) Uncharged tRNA and sensing of amino acid deficiency in mammalian piriform cortex. Science 307, 1776-1778.

89. Maurin AC, Jousse C, Averous J, et al. (2005) The GCN2 kinase biases feeding behavior to maintain amino acid homeostasis in omnivores. Cell Metab 1, 273-277.

90. Cota D, Proulx K, Smith KA, et al. (2006) Hypothalamic mTOR signaling regulates food intake. Science 312, 927-930.

91. Roh C, Han J, Tzatsos A, et al. (2003) Nutrient-sensing mTOR mediated pathway regulates leptin production in isolated rat adipocytes. Am J Physiol Endocrinol Metab 284, E322-E330.

92. Schliess F, Richter L, Vom DS, et al. (2006) Cell hydration and mTOR-dependent signalling. Acta Physiol (Oxf) 187, 223-229.

93. Krause U, Bertrand L, Maisin L, et al. (2002) Signalling pathways and combinatory effects of insulin and amino acids in isolated rat hepatocytes. Eur J Biochem 269, 3742-3750.

94. Anthony JC, Lang CH, Crozier SJ, et al. (2002) Contribution of insulin to the translational control of protein synthesis in skeletal muscle by leucine. Am J Physiol Endocrinol Metab 282, E1092-E1101.

95. Lynch CJ, Patson BJ, Anthony J, et al. (2002) Leucine is a direct-acting nutrient signal that regulates protein synthesis in adipose tissue. Am J Physiol Endocrinol Metab 283, E503-E513.

96. Lynch CJ, Gern B, Lloyd C, et al. (2006) Leucine in food mediates some of the postprandial rise in plasma leptin concentrations. Am J Physiol Endocrinol Metab 291, E621-E630.
97. Christie GR, Hajduch E, Hundal HS, et al. (2002) Intracellular sensing of amino acids in Xenopus laevis oocytes stimulates p70 S6 kinase in a target of rapamycin-dependent manner. $J$ Biol Chem 277, 9952-9957.

98. Yu ZP, Xu M, Liu K, et al. (2014) Leucine markedly regulates pancreatic exocrine secretion in goats. J Anim Physiol Nutr $\mathbf{9 8}$, 169-177.

99. Guo L, Tian HB, Shen J, et al. (2018) Phenylalanine regulates initiation of digestive enzyme mRNA translation in pancreatic acinar cells and tissue segments in dairy calves. Biosci Rep 38, BSR20171189.

100. Liu K, Liu Y, Liu SM, et al. (2015) Relationships between leucine and the pancreatic exocrine function for improving starch digestibility in ruminants. J Dairy Sci $\mathbf{9 8}, 2575-2582$.

101. Tesseraud S, Bigot K \& Taouis M (2003) Amino acid availability regulates $\mathrm{S} 6 \mathrm{~K} 1$ and protein synthesis in avian insulin-insensitive QM7 myoblasts. FEBS Lett 540, 176-180.

102. Yoshizawa F, Hirayama S, Sekizawa H, et al. (2002) Oral administration of leucine stimulates phosphorylation of 4E-BP1 and S6K1 in skeletal muscle but not in liver of diabetic rats. $J$ Nutr Sci Vitaminol (Tokyo) 48, 59-64.

103. Um SH, D'Alessio D \& Thomas G (2006) Nutrient overload, insulin resistance, and ribosomal protein S6 kinase 1, S6K1. Cell Metab 3, 393-402.

104. Kanazawa T, Taneike I, Akaishi R, et al. (2004) Amino acids and insulin control autophagic proteolysis through different signaling pathways in relation to $\mathrm{mTOR}$ in isolated rat hepatocytes. J Biol Chem 279, 8452-8459.

105. Nakashima K, Ishida A, Yamazaki M, et al. (2005) Leucine suppresses myofibrillar proteolysis by down-regulating ubiquitinproteasome pathway in chick skeletal muscles. Biochem Biophys Res Commun 336, 660-666. 